\title{
Accelerating Solutions of Perfect Fluid Hydrodynamics for Initial Energy Density and Life-Time Measurements in Heavy Ion Collisions
}

\author{
T. Csörgő, \\ MTA KFKI RMKI, H-1525 Budapest 114, POBox 49, Hungary and \\ Instituto de Física Teórica - UNESP, Rua Pamplona 145, 01405-900 São Paulo, SP, Brazil \\ M. I. Nagy, and M. Csanád \\ ELTE, Eötvös Loránd University, H - 1117 Budapest, Pázmány P. s. 1/A, Hungary
}

Received on 11 February, 2007

\begin{abstract}
A new class of accelerating, exact, explicit and simple solutions of relativistic hydrodynamics is presented. Since these new solutions yield a finite rapidity distribution, they lead to an advanced estimate of the initial energy density and life-time of high energy heavy ion collisions. Accelerating solutions are also given for spherical expansions in arbitrary number of spatial dimensions.
\end{abstract}

Keywords: Relativistic hydrodynamics; Exact solutions; Initial energy density; Particle spectra and correlations

\section{INTRODUCTION}

Relativistic hydrodynamics can be successfully applied both at the largest and the smallest scales of physics. However, its equations are highly nonlinear, and only few exact relativistic solutions are presently known. We present here a recently found family of exact solutions [1]. These generalize the Landau-Khalatnikov solution [2-4], as they are also accelerating and yield a finite rapidity distribution. They are simple and explicit, similarly to the Hwa-Bjorken solution [5, 6]. We show new $1+3$ dimensional solutions [1], that are reduced to the exact hydro solutions of refs. [7-11] in the accelerationless limit.

\section{NEW SIMPLE SOLUTIONS OF RELATIVISTIC HYDRODYNAMICS}

Let us denote the metric tensor by $g^{\mu \nu}=$ $\operatorname{diag}(1,-1,-1,-1)$, the four-velocity by $u^{\mu}=\gamma(1, v \mathbf{n})$, the pressure by $p$, the energy density by $\varepsilon$, the temperature by $T$, the charged particle density by $n$ and the entropy density by $\sigma$. All fields depend on the coordinates $x^{\mu}$. We denote by $r$ the spatial coordinate $r_{z}$ in $1+1$ dimensions, and the radial coordinate in $1+d$ dimensions (where $r \geq 0$ ). Relativistic hydrodynamics expresses local momentum, energy and charge conservation:

$$
\begin{aligned}
(\varepsilon+p) u^{v} \partial_{v} u^{\mu} & =\left(g^{\mu \rho}-u^{\mu} u^{\rho}\right) \partial_{\rho} p \\
(\varepsilon+p) \partial_{v} u^{v}+u^{v} \partial_{v} \varepsilon & =0 \\
\partial_{v}\left(n u^{v}\right) & =0 .
\end{aligned}
$$

¿From these relations entropy conservation also follows: $\partial_{v}\left(\sigma u^{v}\right)=0$. Our choice of Equations of State (EoS) is that of an ideal gas: $p=n T, \varepsilon=\kappa p$. For an ultra-relativistic ideal gas in $d$ spatial dimensions, $\kappa=d=1 / c_{s}^{2}$. In the considered case, $\sigma \propto T^{d}$.

We use the $\tau$ and $\eta$ Rindler coordinates:

$$
\begin{aligned}
& |r|<|t|: \quad r=\tau \sinh \eta, \quad t= \pm \tau \cosh \eta \\
& |r|>|t|: \quad r= \pm \tau \cosh \eta, \quad t=\tau \sinh \eta
\end{aligned}
$$

In order to generalize the Hwa-Bjorken solution, we introduced the $\lambda>0$ parameter, and found the following class of solutions:

$$
\begin{aligned}
v & =\tanh \lambda \eta \\
p & =p_{0}\left(\frac{\tau_{0}}{\tau}\right)^{\lambda d(1+1 / \kappa)}\left[\cosh \frac{\eta}{2}\right]^{-(d-1) \phi(\lambda)(1+1 / \kappa)}, \\
n & =n_{0}\left(\frac{\tau_{0}}{\tau}\right)^{\lambda d} v(s)\left[\cosh \frac{\eta}{2}\right]^{-(d-1) \phi(\lambda)}, \\
T & =T_{0}\left(\frac{\tau_{0}}{\tau}\right)^{\lambda d / \kappa} \frac{1}{\mathrm{v}(s)}\left[\cosh \left(\frac{\eta}{2}\right)\right]^{-(d-1) \phi(\lambda) / \kappa}
\end{aligned}
$$

The initial pressure, number density and temperature are denoted by $p_{0}, n_{0}$ and $T_{0}$, respectively. The above forms are hydrodynamical solutions for the special values of $\lambda$ and $\kappa$ as given below. The $\lambda=1,|r|<|t|, v(s)=1$ case is the $d$-dimensional generalization of the well known inertial HwaBjorken flow [5, 6], where $\kappa$ and $d>1$ are arbitrary. Here $\mathrm{v}(s)$ is an arbitrary positive scaling function, given by the initial conditions, and $s$ is a scaling variable, that has a vanishing co-moving derivative:

$$
\begin{aligned}
& |r|<|t|: s(\tau, \eta)=\left(\frac{\tau_{0}}{\tau}\right)^{\lambda-1} \sinh [(\lambda-1) \eta], \\
& |r|>|t|: s(\tau, \eta)=\left(\frac{\tau_{0}}{\tau}\right)^{\lambda-1} \cosh [(\lambda-1) \eta],
\end{aligned}
$$

valid for $\lambda \neq 1$. If $\lambda=1$, we have $s=s_{0} \eta$ for $|r|<|t|$, while $s=\tau / \tau_{0}$ for $|r|>|t|$.

The constants $d, \lambda$ and $\kappa$ are constrained: In $1+1$ dimensions, $\lambda$ is arbitrary but $\kappa=d=1$, except if $\lambda=1$ when $\kappa$ is arbitrary. If $d$ is arbitrary, $\lambda=2$ and $\kappa=d$ is a valid solution both for $|r|<|t|$ and $|r|>|t|$, and for $\lambda=1$, we have solution inside the lightcone for arbitrary $\kappa$. Recently, T. S. Biró pointed out [12], that the $\lambda=1 / 2, \kappa=1$ and the $\lambda=3 / 2$, $\kappa=11 / 3$ cases are solutions for $d=3$ dimensional expansions, which can be generalized easily for any $d \in \mathbb{R}$, choosing $\kappa=(4 d-1) / 3$. The function $\phi(\lambda)=0$ for $\lambda=0,1$ or 2 , while it is unity, $\phi(\lambda)=1$ for $\lambda=1 / 2$ or $\lambda=3 / 2$. It is introduced to indicate, that the solutions are dependent on space-time rapidity $\eta$ in the $\lambda=1 / 2$ and $3 / 2$ cases only. The $\lambda \neq 1$ solutions 


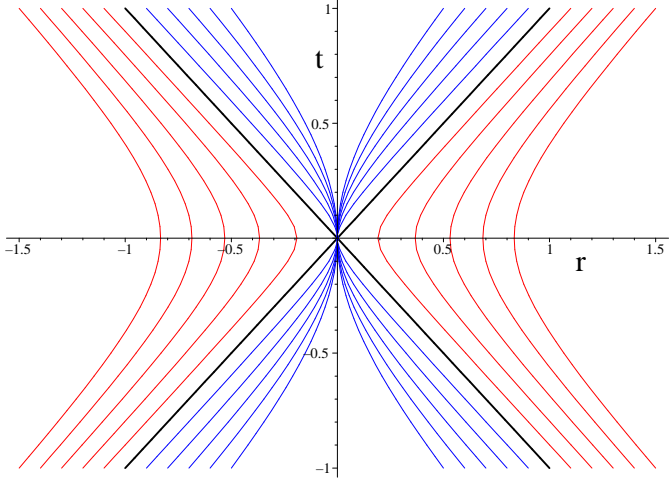

FIG. 1: Fluid trajectories of the $\lambda=2$ exact solution.

describe a flow with relativistic acceleration. If $\lambda=2$, the trajectories are uniformly accelerating, see Fig. 1 and ref. [1].

\section{RAPIDITY DISTRIBUTIONS AND ENERGY DENSITY ESTIMATION}

For $d=1$, the rapidity distribution, $\frac{\mathrm{d} n}{\mathrm{~d} y}$ was calculated in a Boltzmann approximation in ref. [1]. The freeze-out temperature is $T\left(\eta=0, \tau=\tau_{f}\right)=T_{f}$. We assumed [1], that the freeze-out hypersurface is pseudo-orthogonal to $u^{\mu}$. With a saddle-point integration, for $\lambda>0.5$, and $m / T_{f} \gg 1$ (where $m$ is the particle mass), we got

$$
\frac{\mathrm{d} n}{\mathrm{~d} y} \approx n_{f} \tau_{f} \sqrt{\frac{2 \pi m}{T_{f} \lambda(2 \lambda-1)}} \cosh ^{\frac{\alpha}{2}-1}\left(\frac{y}{\alpha}\right) e^{-\frac{m}{T_{f}} \cosh ^{\alpha}\left(\frac{y}{\alpha}\right)},
$$

with $\alpha=\frac{2 \lambda-1}{\lambda-1}$. The "width" of this distribution is

$$
\Delta y^{2}=\frac{\alpha}{m / T_{f}-1 / 2+1 / \alpha} .
$$

The rapidity distribution has a minimum at $y=0$, if $\Delta y^{2}<0$ (i.e. $\left.1 / 2+T_{f} /(4 m)<\lambda<1\right)$, it is flat if $\lambda=1$ or $\lambda=1 / 2+$ $T_{f} /(4 m)$, otherwise it is approximately Gaussian. The typical cases are plotted in Fig. 2.

As an application, we estimate the energy density reached in heavy ion reactions. Let us focus on the thin transverse slab at mid-rapidity, just after thermalization $\left(\tau=\tau_{0}\right)$, illustrated by Fig. 2 of ref. [6]. The radius $R$ of this slab is estimated by the radius of the colliding hadrons or nuclei, and the volume is $d V=\left(R^{2} \pi\right) \tau_{0} d \eta_{0}$. The energy content is $d E=\left\langle m_{t}\right\rangle d n$, where $\left\langle m_{t}\right\rangle$ is the average transverse mass at $y=0$, so similarly to Bjorken, the initial energy density is

$$
\varepsilon_{0}=\frac{\left\langle m_{t}\right\rangle}{\left(R^{2} \pi\right) \tau_{0}} \frac{d n}{d \eta_{0}}
$$

For accelerationless, boost-invariant Hwa-Bjorken flows $\eta_{0}=$ $\eta_{f}=y$, however, for our accelerating solution we have to apply a correction factor of $\frac{\partial \eta_{f}}{\partial \eta_{0}} \frac{\partial y}{\partial \eta_{f}}=\left(\tau_{f} / \tau_{0}\right)^{\lambda-1}(2 \lambda-1)$. Thus
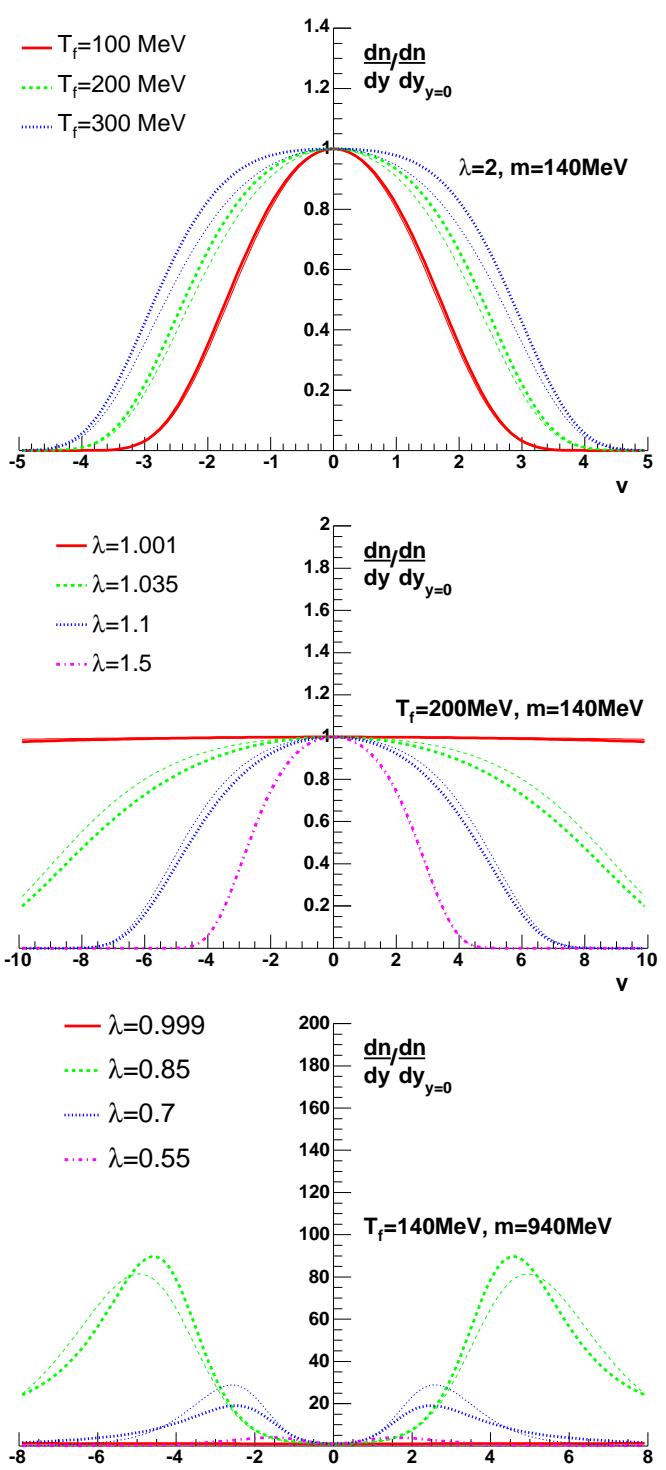

FIG. 2: Normalized rapidity distributions from the new solutions for various $\lambda, T_{f}$ and $m$ values. Thick lines show the result of numerical integration, thin lines the analytic approximation from eq. (12). For $\lambda>1$ and not too big $T_{f}$ it can be used with about $10 \%$ error.

the initial energy density $\varepsilon_{0}$ can be accessed by a corrected estimation $\varepsilon_{c}$ as

$$
\frac{\varepsilon_{c}}{\varepsilon_{B}}=(2 \lambda-1)\left(\frac{\tau_{f}}{\tau_{0}}\right)^{\lambda-1}, \quad \varepsilon_{B}=\frac{\left\langle m_{t}\right\rangle}{\left(R^{2} \pi\right) \tau_{0}} \frac{d n}{d y} .
$$

Here $\varepsilon_{B}$ is the Bjorken estimation, which is recovered if $\frac{\mathrm{d} n}{\mathrm{~d} y}$ is flat (i.e. $\lambda=1$ ), but for $\lambda>1$, both correction factors are bigger than 1 . Hence the initial energy densities are underestimated by the Bjorken formula. Fig. 3 indicates fits to BRAHMS pseudo-rapidity distributions from ref. [13], these fits indicate that $\varepsilon_{c}=8.5-10 \mathrm{GeV} / \mathrm{fm}^{3}$ in $\mathrm{Au}+\mathrm{Au}$ collisions at RHIC. 


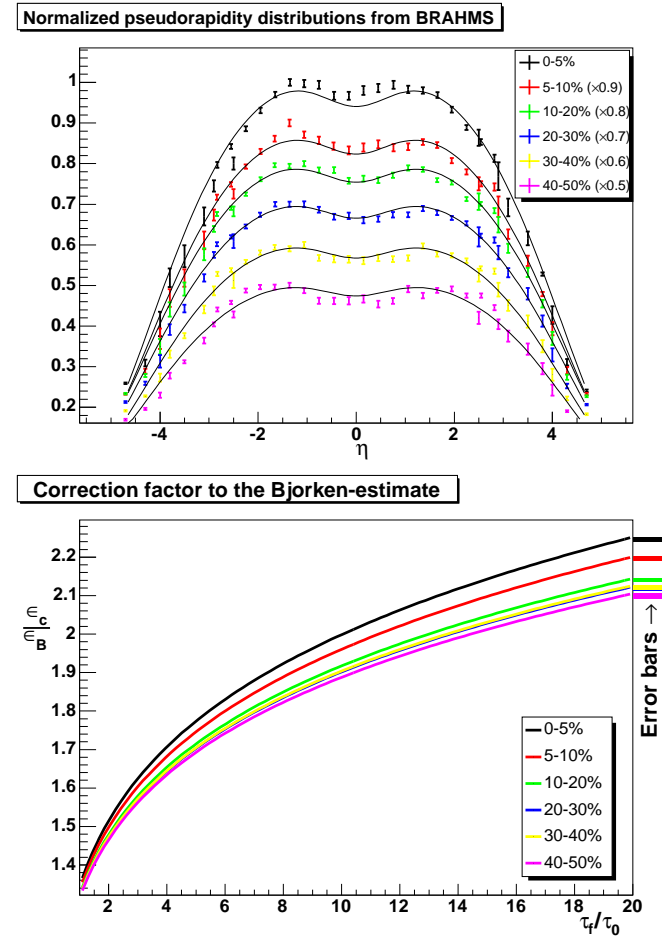

FIG. 3: Top panel: Fits of eq. (12) to $d n / d \eta$ data as measured by the BRAHMS collaboration [13] in $\sqrt{s_{N N}}=200 \mathrm{GeV} \mathrm{Au}+\mathrm{Au}$ collisions at various centralities. Bottom panel: $\varepsilon_{c} / \varepsilon_{B}$ ratio as a function of $\tau_{f} / \tau_{0}$. Using the Bjorken estimate of $\varepsilon_{B}=5 \mathrm{GeV} / \mathrm{fm}^{3}$ as given in the BRAHMS White Paper [14], and $\tau_{f} / \tau_{0}=7-10$, we find an initial energy density of $\varepsilon_{c}=(1.7-2.0) \varepsilon_{B}=8.5-10 \mathrm{GeV} / \mathrm{fm}^{3}$.

\section{LIFE-TIME DETERMINATION}

For a Hwa-Bjorken type of accelerationless, coasting longitudinal flow, Sinyukov and Makhlin [15] determined the longitudinal length of homogeneity as

$$
R_{\text {long }}=\sqrt{\frac{T_{f}}{m_{t}}} \tau_{B}
$$

Here $m_{t}$ is the transverse mass and $\tau_{B}$ is the (Bjorken) freezeout time. However, acceleration influences the estimated lifetimes of the reaction. If the flow is accelerating, the estimated origin of the trajectories is shifted, and we found the following correction of eq. (16), at mid-rapidity, for broad but finite rapidity distribution:

$$
R_{\text {long }}=\sqrt{\frac{T_{f}}{m_{t}}} \frac{\tau_{\mathrm{c}}}{\lambda} \Rightarrow \tau_{\mathrm{c}}=\lambda \tau_{B}
$$

Thus in earlier HBT analyses the effective proper-time duration of the reaction have been underestimated, as pointed out also in refs. [16-19]. BRAHMS pseudo-rapidity distributions in Fig. 3 yield $\lambda \approx 1.2$, and imply a $20 \%$ increase in the estimated life-time of the reaction.

\section{SUMMARY}

We have presented new, simple and accelerating solutions of relativistic hydrodynamics. We have improved quantitatively on Bjorken's initial energy density estimate, by taking into account the longitudinal work, which is important for finite rapidity distributions. We have fitted the BRAHMS pseudo-rapidity distributions with the resulting simple forms, and pointed out that in $\mathrm{Au}+\mathrm{Au}$ collisions at RHIC, $8.5-10$ $\mathrm{GeV} / \mathrm{fm}^{3}$ initial energy densities are reached, a factor of 2 larger, than the Bjorken estimate. We have also corrected the Sinyukov-Makhlin formula for longitudinal work effects, and found an increase of the life-time of the reaction by about 20 $\%$, as extracted from the longitudinal HBT radius parameter in $\mathrm{Au}+\mathrm{Au}$ collisions at $\sqrt{s_{N N}}=200 \mathrm{GeV}$.

Acknowledgements: T. Cs. would like to thank Y. Hama and S. S. Padula for their kind hospitality in Brazil and for their providing an excellent working atmosphere during the ISMD 2006 and WPCF2006 conferences. This work has been supported by the Hungarian OTKA grant T049466, and by a FAPESP grant from São Paulo, Brazil.
[1] T. Csörgó, M. I. Nagy and M. Csanád, nucl-th/0605070.

[2] L. D. Landau, Izv. Akad. Nauk Ser. Fiz. 17 (1953) 51.

[3] I.M. Khalatnikov, Zhur. Eksp. Teor. Fiz. 27 (1954) 529

[4] S. Z. Belenkij and L. D. Landau, Nuovo Cim. Suppl. $3 S 10$ (1956) 15 [Usp. Fiz. Nauk 56 (1955) 309].

[5] R. C. Hwa, Phys. Rev. D 10 (1974) 2260.

[6] J. D. Bjorken, Phys. Rev. D 27 (1983) 140.

[7] T. Csörgó, F. Grassi, Y. Hama and T. Kodama, Phys. Lett. B 565 (2003) 107, [nucl-th/0305059].

[8] T. Csörgô, L. P. Csernai, Y. Hama and T. Kodama, Heavy Ion Phys. A 21 (2004) 73, [nucl-th/0306004].

[9] T. S. Biró, Phys. Lett. B 474, 21 (2000).

[10] T. S. Biró, Phys. Lett. B 487, 133 (2000).

[11] Yu. M. Sinyukov and I. A. Karpenko, Acta Phys.Hung. A25,
141-147 (2006).

[12] T. S. Biró, private communication. The authors are grateful to him for this comment.

[13] I. G. Bearden et al. [BRAHMS Collaboration], Phys. Rev. Lett. 88, 202301 (2002) [nucl-ex/0112001].

[14] I. Arsene et al. [BRAHMS Collaboration], Nucl. Phys. A 757, 1 (2005) [nucl-ex/0410020].

[15] A. N. Makhlin and Yu. M. Sinyukov, Z. Phys. C 39, 69 (1988).

[16] U. A. Wiedemann, Nucl. Phys. A 661, 65C (1999)

[17] T. Renk, Phys. Rev. C 69, 044902 (2004)

[18] M. Csanád, T. Csörgó, B. Lörstad and A. Ster, Nukleonika 49, S49-S55 (2004) [nucl-th/0402037].

[19] S. Pratt, nucl-th/0612010. 